\title{
Galactic substructure and direct detection of dark matter
}

\author{
Marc Kamionkowski* \\ California Institute of Technology, Mail Code 130-33, Pasadena, California 91125, USA
}

Savvas M. Koushiappas ${ }^{+}$

Los Alamos National Laboratory, Mail Stop B227, Los Alamos, New Mexico 87545, USA

(Received 22 January 2008; revised manuscript received 22 March 2008; published 13 May 2008)

\begin{abstract}
We study the effects of substructure in the Galactic halo on direct detection of dark matter, on searches for energetic neutrinos from weakly interacting massive particles (WIMP) annihilation in the Sun and Earth, and on the enhancement in the WIMP annihilation rate in the halo. Our central result is a probability distribution function (PDF) $P(\rho)$ for the local dark-matter density. This distribution must be taken into account when using null dark-matter searches to constrain the properties of dark-matter candidates. We take two approaches to calculating the PDF. The first is an analytic model that capitalizes on the scale-invariant nature of the structure-formation hierarchy in order to address early stages in the hierarchy (very small scales; high densities). Our second approach uses simulation-inspired results to describe the PDF that arises from lower-density larger-scale substructures which formed in more recent stages in the merger hierarchy. The distributions are skew positive, and they peak at densities lower than the mean density. The local dark-matter density may be as small as $1 / 10$ th the canonical value of $\simeq 0.4 \mathrm{GeV} \mathrm{cm}{ }^{-3}$, but it is probably no less than $0.2 \mathrm{GeV} \mathrm{cm}^{-3}$.
\end{abstract}

DOI: 10.1103/PhysRevD.77.103509

PACS numbers: 95.35.+d, 98.35.Gi, 98.35.Pr, 98.62.Gq

\section{INTRODUCTION}

The nature of dark matter remains a mystery. Among the plethora of dark-matter candidates, there are two classes that are sufficiently promising to motivate major experimental searches. The first is a weakly interacting massive particle (WIMP) [1,2], which may arise in supersymmetric extensions of the standard model or in theories that include universal extra dimensions (UED) [3]. The other leading candidate is the axion [4], hypothesized to solve the strong- $C P$ problem.

WIMPs may be detected indirectly through observation of gamma rays or cosmic-ray positrons, antiprotons, and/or antideuterons produced when WIMPs annihilate in the Galactic halo or in the halos of some extragalactic systems. WIMPs might also be detected via observation of energetic neutrinos produced by annihilation of WIMPs that have accumulated in the Sun and/or Earth. There is also an effort to detect dark-matter particles in low-background experiments via detection of the $O(100 \mathrm{keV})$ energy they impart to nuclei from which they elastically scatter. Likewise, dark-matter axions are being sought directly by conversion to photons in resonant-cavity experiments [5]. If dark matter is composed of WIMPs, we may also get some clue to its nature from forthcoming LHC accelerator experiments [6].

Predictions for the event rates for any of these detection schemes depend on the distribution of dark matter in the Galactic halo. The flux of gamma and cosmic rays from

\footnotetext{
*kamion@ tapir.caltech.edu

${ }^{+}$smkoush@lanl.gov
}

WIMP annihilation depends on an integral of the square of the dark matter density over volume, while the rates for energetic neutrinos and direct detection depend only on the local dark-matter density. In early calculations, it was assumed simply that dark matter was smoothly distributed in the Galactic halo, with either an isothermal or NFW [7] radial density profile, and with a local dark-matter density fixed by Milky Way dynamics to be $\rho_{\odot} \simeq 0.4 \mathrm{GeV} \mathrm{cm}^{-3}$.

It has become clear in recent years, from theory and $\mathrm{N}$ body simulations, that the distribution of dark matter in the Galactic halo is not likely to be perfectly smooth, and that some of the dark matter in the Galactic halo will be distributed into subhalos with a variety of sizes [8]. This substructure hierarchy may extend to very small scales [913]. For WIMPs in supersymmetric and UED models, the cutoff scale of the power spectrum is in the range $\left[10^{-6}-10^{2}\right] M_{\oplus}[14,15]$. For axions, the growth of perturbations is suppressed on scales smaller than $\left(m_{a} H\right)^{-1 / 2}$ $[16,17]$, the geometric mean of the inverse of the axion mass and the Hubble constant; for example, for an axion of mass $m_{a} \sim 10^{-5} \mathrm{eV}$, the cutoff scale corresponds to about $10^{-11} M_{\oplus}$.

The implications of such substructure for searches for cosmic rays from WIMP annihilation in the halo have been explored extensively in the literature [18]. Simply stated, if a dark matter halo contains substructure, then the volume integral of the density squared is increased by some boost factor $B$. For example, Ref. [19] recently claimed $B \sim 10^{3}$ enhancements in the direction of the Galactic anticenter, although Ref. [20] finds an enhancement $B \simeq 2-5$. An analytic approach presented in Ref. [21] also finds smaller values of $B$, of order $B \sim 10$. 
The implications of substructure for direct detection of dark matter have, however, been comparatively neglected. This is a serious omission, as substructure implies fluctuations in the local dark-matter density, and these fluctuations imply an uncertainty in the predicted rates for direct-detection experiments and for energetic-neutrino searches. A simple but illustrative example places all of the dark matter in subhalos of density $B$ times the mean density. In this case, the total annihilation rate is enhanced by a boost factor $B$, but the probability that the Solar System lives in such a subhalo is only $B^{-1}$. If $B \gg 1$, this probability is small, implying dire prospects for direct-detection, an alarming conclusion that warrants further investigation.

Few past studies focused on the implications of substructure for the local dark-matter density. Some focused on implications derived from numerical simulations $[22,23]$, thus limited by resolution effects. Others looked at the contribution of tidal streams to the local density $[24,25]$.

The goal of this paper is to begin addressing questions such as: What are the possible values of the local density? How small can they be? What are the most probable values? To answer these questions, we calculate a probability distribution function (PDF) $P(\rho)$ for the local darkmatter density. This PDF accounts for fluctuations in the local density due to substructure. The mean of this distribution is $\simeq 0.4 \mathrm{GeV} \mathrm{cm}^{-3}$, the canonical value determined from dynamics, but we find that the local dark-matter density may be as small as $1 / 10$ th, but probably no less than half this canonical value. The PDF we calculate will have to be convolved with the results of null searches to infer constraints to particle-dark-matter parameters. In addition, the PDF can be used to assess what a substructure enhancement in the halo WIMP annihilation rate implies for the local dark matter density, and vice-versa.

Our first approach, in Sec. II, uses an ansatz about the survival fraction for subhalos that form early at high densities. General arguments will be used to bracket the plausible range of PDFs, and also the plausible lower limit to the local dark-matter density. This analytic approach capitalizes on the scale-invariant nature of the hierarchical formation of galaxies to extrapolate simulation results to the highest-density, smallest-scale substructures that form earliest and that are beyond the reach of simulations. We then perform a second calculation that assumes substructures with NFW profiles are distributed in the Milky Way halo. This second approach describes the local PDF due to larger-scale substructures which have formed from more recent stages in the merger hierarchy, and it thus complements the first approach. In Sec. III, we improve upon this latter calculation by using ingredients on subhalo mass functions and concentration parameters taken from numerical simulations. We compare our work to past literature in Sec. IV, and we state our conclusions in Sec. V.

\section{ANALYTIC MODEL}

In hierarchical structure formation, small halos collapse first, and they then merge to form more massive structures. Because each halo virializes to roughly 200 times the mean cosmological density at collapse, the halo density generally increases with decreasing subhalo mass.

However, a dark matter halo is unlikely to remain completely intact as it merges into larger structures in the hierarchy, and a significant fraction of the mass of each subhalo will be stripped as it is embedded into larger halos. As a result, a significant fraction of the mass of any halo in the hierarchy will be smoothly distributed, rather than contained in substructure. Nevertheless, there will be some fraction that is contained in subhalos of higher density, originating from an earlier stage in the hierarchy, and some fraction of those will be entrapped into even smaller and denser sub-subhalos, etc., all the way down to the smallest scale in the hierarchy. It is important to note that $\mathrm{N}$-body simulations are unlikely to ever be able to resolve the complete hierarchy of substructures, from $10^{12} M_{\odot}$ to $10^{-10} M_{\odot}$, or even smaller, and so some analytic approach is required. Likewise, extended-Press-Schechter calculations of substructure keep track of the most massive subhalo that each mass element occupies, not the least massive subhalo in the hierarchy, which is the step in the hierarchy that determines the local density.

\section{A. Local density probability distribution function}

We describe structure formation as a series of steps in a hierarchy, where the first stages consist of the lowest-mass and densest subhalos, which then merge in subsequent steps in the hierarchy to form more massive structures of lower density. Note that this model simplifies by assuming that each halo has a uniform density. However, as we show below, the qualitative results we obtain are essentially unaltered, at least at high density, if we assume more realistic density profiles, such as isothermal or NFW profiles.

Let $f\left(\rho_{1}\right)$ be the fraction of mass, per logarithmic density interval centered on $\rho_{1}$, that is not yet locked up in halos of higher density. Let $F\left(\rho_{1}\right)$ then be the fraction of the mass today in the Milky Way halo that exists at density greater than $\rho_{1}$. Then $F\left(\rho_{1}\right)$ is related to $f\left(\rho_{1}\right)$ through

$$
\frac{d F}{d \rho_{1}}=-\frac{f}{\rho_{1}}(1-F) .
$$

In other words, $-\left(d F / d \rho_{1}\right) d \rho_{1}$ is the fraction of the mass in the halo today that has a density in the interval $\rho_{1} \rightarrow$ $\rho_{1}+d \rho_{1}$, and this is $f / \rho_{1}$ times $1-F$, the fraction of mass not yet locked up in higher-density subhalos.

Strictly speaking though, $\rho_{1}$ is the density that a halo would have if the mass was uniformly distributed throughout the halo. The true density $\rho$ of the smoothly distributed component of any subhalo is reduced because some of the mass is in subhalos of higher density. A given halo has a 
total mass $M$ and occupies a volume $V=M / \rho_{1}$, but the mass in subhalos is $M F\left(\rho_{1}\right)$, and these subhalos occupy a volume

$$
V_{\mathrm{sub}}=\int_{\rho_{1}}^{\rho_{\max }} d \rho_{1}^{\prime}\left(-d F / d \rho_{1}^{\prime}\right)\left[M / \rho\left(\rho_{1}^{\prime}\right)\right] .
$$

The smoothly distributed matter has mass $M\left[1-F\left(\rho_{1}\right)\right]$, and it occupies a volume $V-V_{\text {sub }}$, and so the true density of the smoothly distributed component of a halo of mean density $\rho_{1}$ is

$$
\rho\left(\rho_{1}\right)=\rho_{1} \frac{1-F\left(\rho_{1}\right)}{1+\rho_{1} \int_{\rho_{1}}^{\rho_{\max }} \frac{d F}{d \rho_{1}^{\prime}} d \rho_{1}^{\prime} \frac{1}{\rho\left(\rho_{1}^{\prime}\right)}} .
$$

Thus, for example, locally in the Milky Way, the density $\rho_{\odot} \simeq 0.4 \mathrm{GeV} \mathrm{cm}^{-3}$ is the value determined from dynamics, but $\rho$, the smoothly-distributed component, may be smaller, reduced because some of the mass is in subhalos.

Equation (3) is an integral equation for $\rho\left(\rho_{1}\right)$. To solve it, we differentiate the expression for $\rho\left(\rho_{1}\right)$ with respect to $\rho_{1}$ to obtain a differential equation,

$$
\frac{d \rho}{d \rho_{1}}=\frac{\rho^{2}}{\rho_{1}^{2}\left[1-F\left(\rho_{1}\right)\right]} .
$$

This can then be integrated to obtain

$$
\rho\left(\rho_{1}\right)=\left\{\int_{\rho_{1}}^{\rho_{\max }} \frac{d \rho_{1}^{\prime}}{\left(\rho_{1}^{\prime}\right)^{2}\left[1-F\left(\rho_{1}\right)\right]}\right\}^{-1} .
$$

This result could have also been obtained simply by discretizing and summing the contributions of each density interval to the volume.

To recapitulate, we postulate a survival fraction $f\left(\rho_{1}\right)$ for halos of mean density $\rho_{1}$. We obtain the fraction of the Milky Way mass smoothly distributed in halos of mean density $\rho_{1}$ by solving Eq. (1). We then obtain the true density $\rho$ of the smoothly distributed component of halos of density $\rho_{1}$ from Eq. (5). The fraction of mass in the Milky Way with density in the interval $\rho \rightarrow d \rho$ is then $(-d F / d \rho)=\left(-d F / d \rho_{1}\right)\left(d \rho / d \rho_{1}\right)^{-1}$. The final step is then to simply note that the density probability distribution function $P(\rho)$ that we seek is actually the fraction of the Milky Way volume, rather than mass, at density $\rho$, and this is

$$
P(\rho)=\frac{1}{V} \frac{d V}{d \rho}=\frac{(1 / \rho)(-d F / d \rho)}{\int_{\rho_{\odot}}^{\rho_{\max }} d \rho_{1}^{\prime}\left[1 / \rho\left(\rho_{1}^{\prime}\right)\right]\left(-d F / d \rho_{1}^{\prime}\right)} .
$$

With this result, we have mapped the problem of determining $P(\rho)$ to the problem of determining the survival fraction $f\left(\rho_{1}\right)$. In principle, $f(\rho)$ can be determined with simulations, but the appropriate simulations have not yet been performed. Thus, here we will make some educated guesses, based partially on estimates from existing simulations, and then explore the implications of these guesses for $P(\rho)$.
N-body simulations of cosmological formation of Milky-Way-like halos suggest that only about [10-20]\% of the Milky-Way-halo mass is in its 10 largest progenitors [26]. This fraction could potentially be larger as halos in future numerical simulations get more highly resolved. For example, the survival fraction of early forming subhalos (thus tightly bound) is higher ( $\sim 40 \%$ of their mass survives [12]) than recently forming subhalos; therefore if subhalos are resolved at higher densities, the fraction of mass in substructure as measured in simulations could increase. If we assume [10-40]\% of the Milky Way halo mass is in subhalos with a density $\sim 1 / 10$ th that of the mean density of the Milky Way, then this implies very roughly that $f\left(\rho_{1}\right) \simeq 0.04-0.16$, at least for the most recent stage in the hierarchy $\left(\rho_{1} \simeq \rho_{\odot}\right)$. Therefore, for the remainder of this calculation we will consider a range $f\left(\rho_{\odot}\right)=$ $0.05-0.2$.

How then does $f\left(\rho_{1}\right)$ vary for higher masses? Before answering, we first note that the density and mass of the inner $10 \mathrm{kpc}$ of the Milky Way, where we live, suggest a formation redshift $z \sim 10$ for the inner $10 \mathrm{kpc}$ of the Milky Way halo. At these and higher redshifts, the Universe is Einstein-de Sitter. Moreover, the effective spectral index for primordial perturbations spans the range $-2.6 \lesssim n_{\text {eff }} \lesssim-2.1$ for mass scales $10^{11} M_{\odot} \gtrsim M \gtrsim$ $10^{-6} M_{\oplus}$, corresponding to the range of substructure mass scales we are considering here. If we approximate $n_{\text {eff }} \simeq$ constant, then in the appropriate range of stages in the hierarchy, structure formation is scale invariant. One reasonable guess is thus that $f\left(\rho_{1}\right)=$ constant, that the hierarchy of substructures in the Galactic halo is scale invariant.

However, what is more likely, for several reasons, is that $f\left(\rho_{1}\right)$ decreases with increasing $\rho_{1}$. First of all, subhalos in the earlier stages of the hierarchy undergo more orbits in their parent halos between the time they form and today, and this increases the efficiency with which they will be stripped [20]. Second, smaller subhalos may be disrupted by tidal interactions with stars [27]. Third, the effective spectral index $n_{\text {eff }} \rightarrow-3$ for earlier stages in the hierarchy. Thus, earlier in the hierarchy there is less time for a subhalo to virialize fully before it becomes enveloped by the next larger halo in the hierarchy. To model these effects, we thus consider $f\left(\rho_{1}\right)=f\left(\rho_{\odot}\right)\left(\rho_{1} / \rho_{\odot}\right)^{-\alpha}$ with $\alpha>0$.

The differential equation in Eq. (1) is readily solved for these $f\left(\rho_{1}\right)$ parametrizations. For $f\left(\rho_{1}\right)=$ constant, $F(\rho)=1-\left(\rho_{1} / \rho_{\text {max }}\right)^{f}$. For example, if we take $\rho_{\text {max }} / \rho_{\odot}=1000$, then for $f\left(\rho_{1}\right)=0.05,0.1$, or 0.2 , we find that in the Milky Way, $F\left(\rho_{\odot}\right)=0.29,0.5$, and 0.75, respectively; the remaining $(1-F)$ mass is in the smooth component. Thus, if $f=0.1$, then half of the mass of the Milky Way halo at radii $r \sim 8 \mathrm{kpc}$ is smoothly distributed, and the other half is in subhalos. A larger $f$ reduces the smooth component, as does a larger $\rho_{\max }$. As we show 
below, a power-law $f\left(\rho_{1}\right)$ increases the smooth component. The density of the smooth component can be estimated to be $(1-F) \rho_{\odot}$. More precisely, the solution of Eq. (4) gives the density of the smooth component as $\rho\left(\rho_{\odot}\right)=0.74,0.55$, and 0.3 , respectively.

For a power-law $f\left(\rho_{1}\right)=f\left(\rho_{\odot}\right)\left(\rho_{1} / \rho_{\odot}\right)^{-\alpha}($ and $\alpha>0)$,

$$
F\left(\rho_{1}\right)=1-\exp \left\{-\frac{f}{\alpha}\left[1-\left(\frac{\rho_{1}}{\rho_{\max }}\right)\right]\right\},
$$

and if $\alpha$ is not too small ( $\alpha \geq 0.5$ ), the dependence on the cutoff density $\rho_{\max }$ disappears. In that case, $F\left(\rho_{\odot}\right) \simeq 1-$ $\exp \left[-f\left(\rho_{\odot}\right) / \alpha\right] \simeq f\left(\rho_{\odot}\right) / \alpha$, where the last approximation valid for $f\left(\rho_{\odot}\right) \ll \alpha$. One guess for $f\left(\rho_{1}\right)$ is that it is inversely proportional to the formation time $t \propto \rho^{-1 / 2}$ for halos of density $\rho$. If so, then $F\left(\rho_{\odot}\right) \simeq 2 f\left(\rho_{\odot}\right)$ is the fraction of the Milky Way mass that is in subhalos, roughly $20 \%$ for $f\left(\rho_{\odot}\right)=0.1$. A stronger dependence on $\rho_{1}$ would result in a smaller $F\left(\rho_{\odot}\right)$. It is then straightforward to calculate the PDF $P(\rho)$ to find power-law dependence $P(\rho) \propto \rho^{-(2+\alpha)}$ for densities above the smooth density.

\section{B. Results and discussion}

To illustrate, Fig. 1 shows the PDFs, on a log-log plot, for four combinations of the parameters $f\left(\rho_{\odot}\right)$ and $\alpha$ in the model. The distributions feature a high-density tail (close

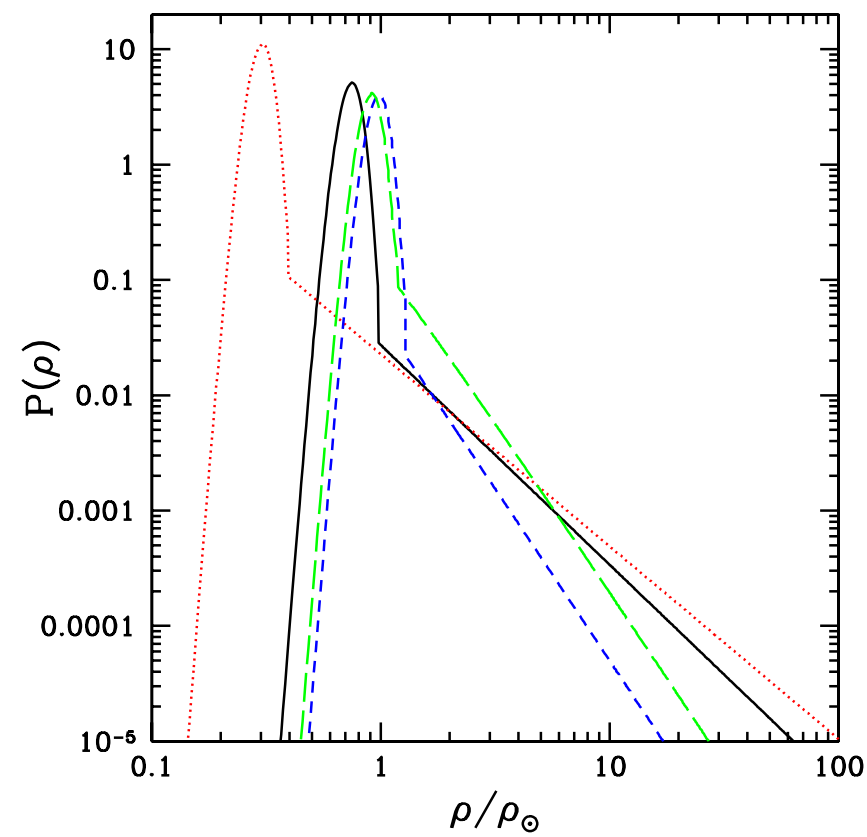

FIG. 1 (color online). The local dark-matter-density probability distribution function $P(\rho)$ for the analytic model, as a function of the density $\rho$ scaled by the mean density $\rho_{\odot}$, for $\left\{f\left(\rho_{\odot}\right), \alpha\right\}=\{0.05,0\}$ (black solid curve), $\{0.2,0\}$, (dotted red curve), $\{0.05,1\}$, (short-dash blue curve) $\{0.2,1\}$ (long-dash green curve). We smooth the Dirac delta function for the smooth component to a Gaussian of rms one-tenth the smoothcomponent density. The power-law tails are due to subhalos. to a power-law), due to the fraction $(1-F)$ of the mass that is in substructure. Also shown is a smooth component at a density $\rho_{\mathrm{sm}}<\rho_{\odot}$; for purposes of illustration, we smooth the Dirac delta-function dependence of this smooth component to a Gaussian of rms one-tenth the smoothcomponent density. The Figure shows that as $f\left(\rho_{\odot}\right)$ is increased, the amplitude of the high-density tail is increased at the expense of the smooth component. We also see that increasing $\alpha$ increases the smooth-component density while decreasing the amplitude of the high-density tail.

Table I provides numerical results for the smoothcomponent density, the fraction of the volume occupied by the smooth component, and the annihilation enhancement $B$ (discussed in more detail below) for PDFs $P(\rho)$ parametrized by the survival fraction $f\left(\rho_{\odot}\right)$ and the survival-fraction power-law index $\alpha$.

Here are some comments and general conclusions from these results so far:

(1) If $f\left(\rho_{\odot}\right)$ is roughly $f \lesssim 0.2$ as suggested by numerical simulations, then the reduction in the smoothcomponent density is no less than $30 \%$ the mean density. The implied fractional uncertainty in the local dark-matter density is thus not too much larger than that (roughly factor of 2) implied by uncertainties in the stellar/gas contribution to the local rotation curve, or the uncertainties that arise if we allow for a flattened halo (which generally increase the local dark-matter density).

(2) The fraction of the volume occupied by the smooth component is larger than the fraction of the mass in the smooth component, as the higher-density components occupy correspondingly less volume. As a result, in most of the models, the density in the vast majority of the halo volume is the density of the smooth component.

(3) We have assumed that each halo and subhalo has a uniform density. More realistically, the density of

TABLE I. The density $\rho_{\text {smooth }}$ of the smooth component, the fraction of the volume occupied by the smooth component, and the annihilation enhancement $B$ for density distributions $P(\rho)$ parameterized by the survival-fraction amplitude $f\left(\rho_{\odot}\right)$ and the survival-fraction power-law index $\alpha$.

\begin{tabular}{lllcc}
\hline \hline$f\left(\rho_{\odot}\right)$ & $\alpha$ & $\rho_{\text {smooth }}$ & smooth fraction & $B$ \\
\hline 0.05 & 0 & 0.75 & $95 \%$ & 47 \\
0.1 & 0 & 0.56 & $91 \%$ & 88 \\
0.2 & 0 & 0.3 & $83 \%$ & 156 \\
0.05 & 0.5 & 0.95 & $97 \%$ & 3.9 \\
0.1 & 0.5 & 0.89 & $94 \%$ & 6.8 \\
0.2 & 0.5 & 0.78 & $88 \%$ & 12 \\
0.05 & 1 & 0.98 & $98 \%$ & 1.3 \\
0.1 & 1 & 0.96 & $95 \%$ & 1.6 \\
0.2 & 1 & 0.91 & $91 \%$ & 2.1 \\
\hline \hline
\end{tabular}


each halo and subhalo will decrease with radius, perhaps with an NFW profile, which has a density that depends on radius $r$ as $\rho \propto r^{-1}$ in the inner regions. The volume in such a halo changes with density as $(d V / d \rho) \propto \rho^{-3}$ for $\rho \rightarrow \infty$. This falls with density more rapidly at large $\rho$ than $P(\rho) \propto$ $\rho^{2+\alpha}$ as long as $\alpha<1$. Thus, if $\alpha<1$, the highdensity scaling of our $P(\rho)$ will be unaltered by convolving our halo density distribution with NFW profiles.

By contrast, this analytic model is weakest perhaps at low densities (larger-scale substructures), where details of the low-density outer regions of merging substructures may be important in determining the local density, the density of what we have called the smooth component. To address this shortfall, we continue in the next subsection to calculate the halo PDF in a different model, and one that will then preview the numerical results that we present later.

\section{Discrete subhalo populations}

In this section we consider another illustrative model, one in which earlier generations of subhalos are subsumed and then smoothly distributed in radius into their larger host halos. In this case, the Milky Way halo is seen as an amalgamation of smaller halos, distributed in mass with some mass function $d n / d M$ (e.g., a Press-Schechter-like mass function, or one taken from simulations). Furthermore, each of these subhalos has an NFW density profile. In this scenario, there is no smooth component of dark matter distributed in the Milky Way halo; instead, the whole halo is made up of adjacent individual subhalos. Although there may be a wide variety of halo masses in this scenario, they all have comparable mean densities, and mean densities comparable to the Milky Way density. If this is the case, then the subhalos fill the entire volume of the Milky Way. Such a scenario neglects the halos-in-halos problem, but it may more accurately describe the PDF due to larger-scale substructures, which arise from the most recent stages in the merger hierarchy.

Consider now the volume of radius $R$ occupied by one of these subhalos. If the Milky Way mass were uniformly distributed, the matter in this volume would have some uniform density $\bar{\rho}$. However, the subhalo has its own NFW density profile,

$$
\rho(r)=\frac{\rho_{s}}{\left(r / r_{s}\right)\left(1+r / r_{s}\right)^{2}},
$$

where $r_{s}$ is the scale radius. This scale radius is related to the concentration parameter $c_{\mathrm{v}}$ through $R=c_{\mathrm{v}} r_{s}$; i.e., halos with larger $c_{\mathrm{v}}$ are more centrally concentrated. The characteristic density $\rho_{s}$ is related to $\bar{\rho}, R$, and $c_{\mathrm{v}}$ through $\bar{\rho}=3 \rho_{s} f\left(c_{\mathrm{v}}\right) / c_{\mathrm{v}}^{3}$, where $f\left(c_{\mathrm{v}}\right)=\ln c_{\mathrm{v}}-c_{\mathrm{v}} /\left(1+c_{\mathrm{v}}\right)$.

The fraction of the volume at density $\rho$ in this model is $P(\rho)=(1 / V)(d V / d \rho)=\left(3 / R^{3}\right) r^{2}(d r / d \rho)$. It can be cal-

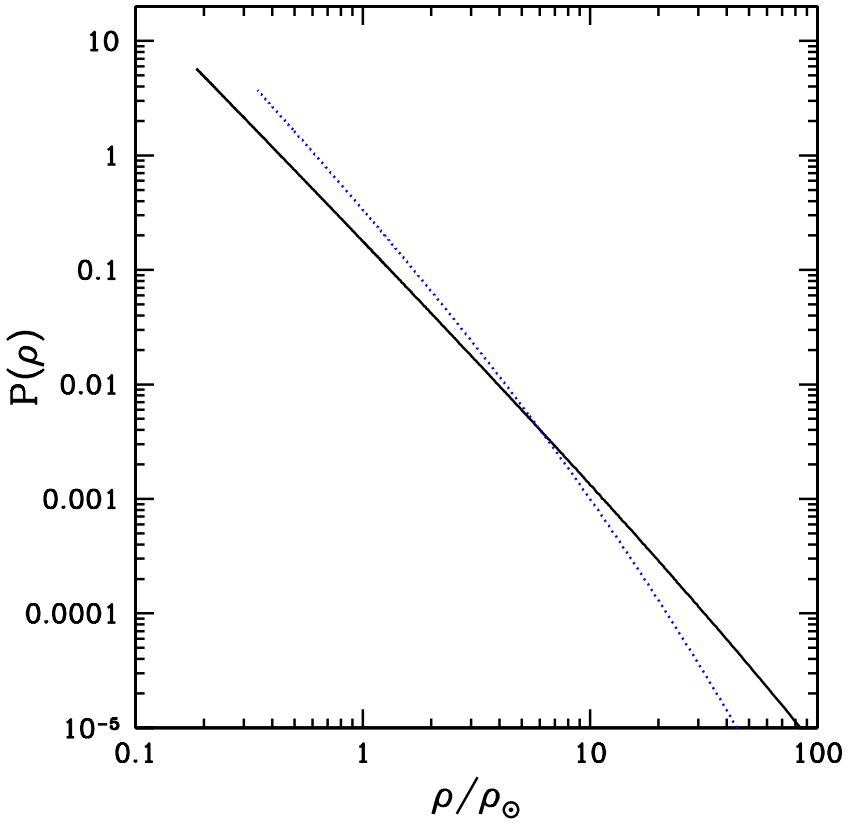

FIG. 2 (color online). The local dark-matter-density probability distribution function $P(\rho)$, for the discrete-subhalo model, as a function of the density $\rho$ scaled by the mean density $\rho_{\odot}$, for $c_{\mathrm{v}}=10$ (black solid curve) and $c_{\mathrm{v}}=2$ (dotted red curve).

culated analytically, but the expressions are algebraically unwieldy and unilluminating. The important thing is that $(d V / d \rho) \propto \rho^{-3}$ for $\rho \gg \rho_{s}$ and $(d V / d \rho) \propto \rho^{-2}$ for $\rho \ll$ $\rho_{s}$. The results for $P(\rho)$ in this model are shown in Fig. 2. These models predict a broad range of $\rho$, but the median densities are $\rho_{\text {med }}=0.35 \rho_{\odot}$ and $0.58 \rho_{\odot}$ for $c_{\mathrm{v}}=10$ and 2 , respectively. The $95 \%$ C.L. lower limits to $\rho$ are $\rho_{95}=$ $0.20 \rho_{\odot}$ and $0.36 \rho_{\odot}$ for $c_{\mathrm{v}}=10$ and 2 , respectively.

Note that nowhere in this discussion did we specify the subhalo mass or mass function. The results apply as long as all of the subhalos have the same concentration parameter and virial density, independent of their mass. More realistically, there will be a range of concentration parameters and virial densities, and this will be explored in the next section. Note also that we have assumed no smooth component, but more realistically the outer parts of each subhalo will be tidally stripped to provide a smooth component. This can be seen roughly by noting that the density of the NFW profiles at the maximum radius is roughly $0.19-0.34$ the mean density. This will also be explored in the next section.

\section{Annihilation enhancement due to substructure}

As we mention in the Introduction, substructure in the halo implies an enhancement to the dark-matter annihilation rate. For example, a $10^{-4} M_{\oplus}$ halo has a characteristic density which is roughly 1000 times the local dark-matter density. If all the dark matter in the halo today resided in such subhalos, then the annihilation flux would be boosted 
by a factor of 1000 . However, the probability for the Solar System to be in such a subhalo would then be only $0.1 \%$.

The analytic models developed here allow us to evaluate the enhancement of the annihilation rate due to substructure and relate it to the local dark-matter density. The enhancement in the annihilation rate over the smooth dark-matter distribution is

$$
B=\frac{\int \rho^{2} d V}{\int \rho_{\odot}^{2} d V}=\int P(\rho) \frac{\rho^{2}}{\rho_{\odot}^{2}} d \rho .
$$

If the survival fraction $f\left(\rho_{1}\right)$ has a power-law dependence $f\left(\rho_{1}\right) \propto \rho_{1}^{-\alpha}$, then there will be a contribution $\sim f\left(\rho_{\odot}\right)\left(\rho_{\max } / \rho_{\odot}\right)^{1-\alpha} /(1-\alpha)$ to the enhancement from the high-density tail in $P(\rho)$. Thus, a large annihilation enhancement requires $\alpha<1, f\left(\rho_{\odot}\right)$ not too small $\left(f\left(\rho_{\odot}\right) \geq 0.2\right)$, and $\rho_{\max } \gg \rho$. For example, we estimate that for $f(\rho)=0.2$ (a constant) and $\rho_{\max } \simeq 1000 \rho_{\odot}$, the enhancement factor will be $B \sim 200$. The enhancement factor will be roughly proportional to $f\left(\rho_{\odot}\right)$, and it will decrease, possibly sharply, as $\alpha$ is increased from zero. Our numerical results find that $B$ decreases to values $B \leqslant 10$ for $\alpha=0.5$ and $f\left(\rho_{\odot}\right) \lesssim 0.2$. It is also important to note that if the power-law exponent of $\alpha$ is such as to allow a large enhancement $B$, then the value of that enhancement is likely to depend strongly on the cutoff density $\rho_{\max }$.

For the case where the Milky Way halo is composed of discrete subhalos with no smooth component, the annihilation rate in this halo is enhanced over that in the uniformdensity halo by a factor

$$
B\left(c_{\mathrm{v}}\right)=\frac{4 \pi \int r^{2} d r[\rho(r)]^{2}}{4 \pi \int r^{2} d r \bar{\rho}^{2}}=\frac{1}{3} \frac{c_{\mathrm{v}}^{3} g\left(c_{\mathrm{v}}\right)}{\left[f\left(c_{\mathrm{v}}\right)\right]^{2}},
$$

where $g\left(c_{\mathrm{v}}\right)=(1 / 3)\left[1-\left(1+c_{\mathrm{v}}\right)^{-3}\right]$. This enhancement factor is $B\left(c_{\mathrm{v}}=1\right) \simeq 2.6$, it grows to $B\left(c_{\mathrm{v}}=10\right) \simeq 50$, and grows roughly as $c_{\mathrm{v}}^{3} / 9\left[\ln c_{\mathrm{v}}\right]^{2}$ for $c_{\mathrm{v}} \gg 1$, in broad agreement with the results presented in Ref. [21].

In summary, we conclude that:

(1) Very large (i.e., $\gg 10$ ) enhancements to the annihilation rate require a survival fraction $f$ that is roughly constant with density. In other words, the survival fraction for a halo must be largely independent of its formation time. If earlier halos are less likely to survive, then a large annihilation enhancement requires a survival fraction today that is large, and perhaps too large to be consistent with numerical simulations of halo formation. This is consistent with Ref. [20], who claim that survival fractions of the earliest halos are $\sim 0.1 \%-0.5 \%$ and an annihilation fraction $B \simeq 2-5$.

(2) The annihilation enhancement generally increases at the expense of the local density. For example, in the $\alpha=0$ model, an annihilation enhancement $B \approx$ 150 implies a reduction by a factor of 3 in the local dark-matter density. The annihilation enhancement will generally increase and the local smooth component will also generally decrease for larger $\rho_{\max }$.

(3) Strictly speaking, the PDF derived here is for the dark-matter density locally (or at some other specified point), and the annihilation enhancement $B$ is the enhancement in the annihilation rate in a local volume. It is expected that the PDF, as well as $B$, will vary with radius in the Galactic halo. Since the central higher-density regions of the halo presumably formed earlier, the amount of substructure should be reduced there (i.e., a larger smooth fraction and smaller $B$ ), and conversely for larger radii (smaller smooth fraction and larger $B$ ). These trends are consistent with the enhancement factors found, for example, in Ref. [19], which calculate the annihilation intensities as a function of observation direction.

\section{SIMULATION-INSPIRED RESULTS}

We now proceed to build on the analytic calculation in the previous section by implementing a substructure fraction, a subhalo mass function, and a range of concentration parameters taken from simulations.

We assume that when averaged over time, the solar neighborhood has a mean density $\rho_{\odot} \approx 0.4 \mathrm{GeV} \mathrm{cm}^{-3}$. In a halo that contains substructure, this density can be split into a smooth component, and a component that arises from the presence of subhalos,

$$
\rho_{\odot}=\rho_{\mathrm{sm}}+\rho_{\mathrm{sub}}=\rho_{\mathrm{sm}}+\int_{\epsilon}^{\eta} M \frac{d n}{d \ln M} d \ln M .
$$

Here, $d n / d \ln M$ is the number density of objects per logarithmic mass interval, and the integral is performed over a mass range, $\epsilon \leq M \leq \eta \leq M_{\mathrm{MW}}$. Strictly speaking, the mass function depends on the primordial power spectrum and upon the physics of halo merging and stripping of halos.

Numerical simulations find that the mass function can be approximated over a wide mass range by a power law, $d n / d \ln M \sim M^{-\beta}$, with $\beta \sim 0.9$ [12,28,29], with indications that a similar form remains down to subsolar subhalos $[20,30]$. We normalize the mass function so that a fraction $\xi$ of the local mean dark-matter density is in objects with masses between $\epsilon \approx 10^{-10} M_{\odot}$ and $\eta \approx 10^{12} M_{\odot}$. This implies that the value of the smooth component is $\rho_{\mathrm{sm}}=$ $(1-\xi) \rho_{\odot}$. The upper limit $\eta$ of the integration must always be less than the mass of the Milky Way. However, the lower integration limit $\epsilon$ depends on the physics of the dark-matter particle and on small-scale structure assemblage.

We model each subhalo with a two-parameter NFW profile, taking the two parameters to be the mass and the concentration parameter, and using a virial radius (maximum radius) defined by assuming the density of each halo 
is 200 times the matter density at the redshift of formation. Each subhalo is then assigned a concentration that is selected from a $P\left(c_{\mathrm{v}}\right)$ log-normal distribution about a mean determined by the value of $\sigma(M)$ and the evolution of linear perturbations, and with a scatter $\sigma\left[\log \left(c_{\mathrm{v}}\right)\right]=$ 0.14 inferred from numerical simulations [31,32]. In this model, the mean concentration is a weak function of mass, $c_{\mathrm{v}} \sim M^{-\gamma}$, where $\gamma \approx 0.13$ for scales near $M_{\star}$ at $z \approx 0$, and scales as $c_{\mathrm{v}} \approx 33\left(M / 10^{8} M_{\odot}\right)^{-0.06}$ for halos with masses $M \leq 10^{8} M_{\odot}$.

We calculate the density probability distribution function as

$$
P(\rho)=\int_{\epsilon}^{\eta} \frac{d n}{d \ln M} \frac{d v(M, \rho)}{d \rho} d \ln M .
$$

Here, $d v(M, \rho) / d \rho=\int\left[d v\left(M\left(c_{\mathrm{v}}\right)\right) / d \rho\right] P\left(c_{\mathrm{v}}\right) d c_{\mathrm{v}}$, where $v(M, \rho)=4 \pi\left(r_{\mathrm{s}} \tilde{r}_{c}\right)^{3} / 3$ is the volume in a halo of mass $M$, where $\tilde{r}_{c}$ is obtained by solving

$$
\tilde{r}_{c}\left(1+\tilde{r}_{c}\right)^{2}=\rho_{\mathrm{s}} / \rho
$$

In solving Eq. (13), we assume that halos can only have a finite size, given by $\tilde{r}_{\max }=c_{\mathrm{v}}$. If the solution is such that $\tilde{r}<c_{\mathrm{v}}$, then the volume with density greater than $\rho$ is simply $4 \pi r_{\mathrm{s}}^{3} \tilde{r}^{3} / 3$, while if $\tilde{r}>c_{\mathrm{v}}$, then the volume is given by $4 \pi r_{\mathrm{s}}^{3} c_{\mathrm{v}}^{3} / 3$. However, in order to approximate the effects of tidal interactions, Eqs. (12) and (13) are always solved for $\rho>\rho_{\text {sm }}$; i.e., we assume the tidal radius of each subhalo is defined as the radius where the density of the halo is equal to the local smooth density component.

Figure 3 shows the density probability distribution function as a function of $\rho$, expressed as a fraction of $\rho_{\odot}$. Different line types depict the PDFs that corresponds to $\xi=0.1,0.5$, and 0.8 . As before, we smooth the Dirac delta function at the value of the smooth density by a Gaussian with an RMS one-tenth the smooth component density. The range that corresponds to the subhalo population for each $\xi$ represents the spread in $P(\rho)$ that arises from a spread in the lower integration limit $\epsilon$ and in the power-law exponent $\beta$ of the subhalo mass function. More precisely, for each value of $\xi$, the upper curve corresponds to a subhalo power-law index $\beta=1.1$ and integration limits $\epsilon=10^{-10} M_{\odot}$ and $\eta=10^{-9} M_{\odot}$; i.e. a steep subhalo mass function with most of the population in objects of extremely small mass. The lower curve is obtained by flattening the subhalo mass function to a power-law exponent $\beta=0.7$ and integration limits $\epsilon=10^{10} M_{\odot}$ (since we know the LMC exists) and $\eta=10^{12} M_{\odot}$, roughly speaking the Milky Way mass; i.e., this is a model with only very massive substructure.

The value of $\xi$ fixes the value of $\rho_{\mathrm{sm}}$, and densities $\rho>$ $\rho_{\text {sm }}$ come from subhalos. The slope of $P(\rho)$ is set in Eq. (12) by the slope of $v(\rho)$. At densities where $\tilde{r} \ll 1$, the volume within which the density is greater than a particular value is $v \sim \tilde{r}^{3} \sim \rho^{-3}$. For lower densities, prob-

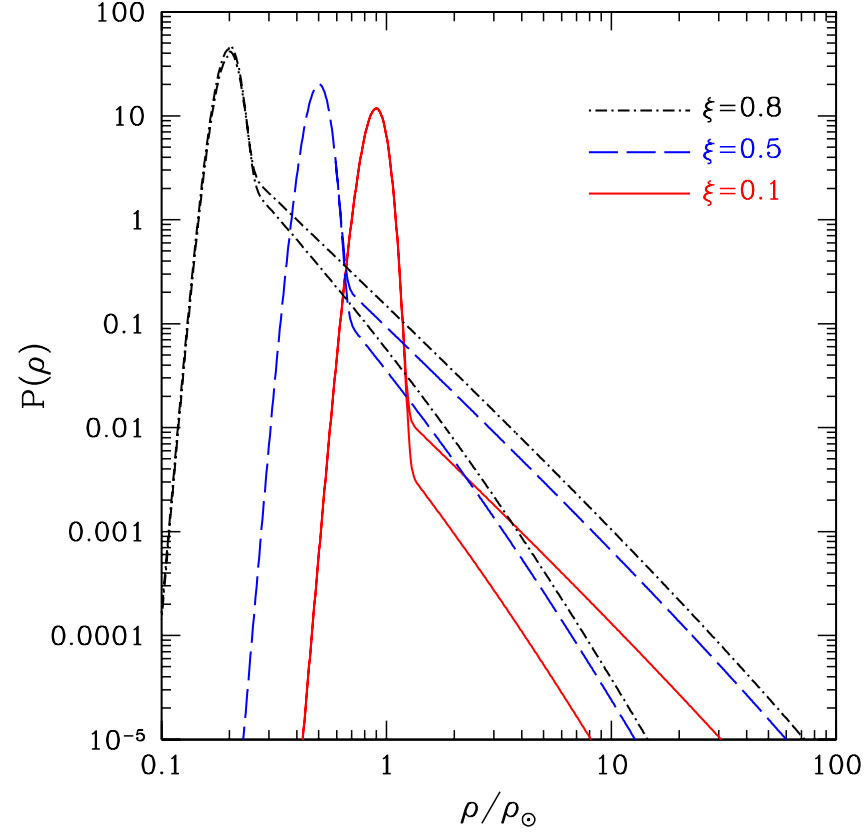

FIG. 3 (color online). The probability distribution function in the solar neighborhood for the simulation-inspired calculation. The solid, long-dash and dot-dash curves correspond to $\xi=0.1$, 0.5 , and 0.8 , respectively. As in Fig. 1, we smooth the Dirac delta function for the density value of the smooth component with a Gaussian of rms a tenth of the smooth-component density. The upper and lower curves for each value of $\xi$ show the range of contribution of the subhalo population that arises from uncertainties in the subhalo mass function, as well as the subhalo population (see text).

ing the outer regions of a halo-i.e., $\tilde{r} \gg 1$-the volume scales as $v \sim \tilde{r}^{3} \sim \rho^{-1}$. Thus, we expect a slope for $P(\rho)$ of -2 for low densities, and a slope of -4 as the density increases.

The sharp transition between the smooth component and the subhalos in Fig. 3 results from the assumption of a pure power law for the dark-matter profile in subhalos [7]. In reality, the transition will be much more gradual, reflecting the fact that subhalos are embedded in the potential well of their host Milky Way halo. As $\xi \rightarrow 1$, the smooth component approaches zero. There is still a lower limit, $\rho \gtrsim$ $0.04 \mathrm{GeV} \mathrm{cm}^{-3}$, attained at a value $\xi \gtrsim 0.92$, to the local halo density that arises from the overlap of the outskirts of subhalos. These calculations thus imply that there is significant probability for the local halo density to be below $\rho_{\odot}$, but also that there is likely a minimum possible value to the local density. Simulations (e.g., Ref. [11]) suggest a value $\xi \simeq 0.5$, but further investigation is required to determine this important parameter more precisely and robustly.

The annihilation enhancement in this approach can be estimate as in Ref. [21]. Namely, in a halo of mass $M$ and with a cutoff in the subhalo mass function at $m_{0}$, the boost factor is the solution of an integral equation that takes into 
account the halo-in-halo problem, and is approximated by $B \approx 0.1\left[\left(M / m_{0}\right)^{0.13}-1\right]$. For a cutoff in the power spectrum at microhalo scales, $m_{0} \approx 10^{-6} M_{\odot}$, and a Milky Way mass of $M \approx 10^{12} M_{\odot}, B \approx 20$. The weak dependence of the boost factor to the cutoff scale of the subhalo mass function is an outcome of the flatness of the dark-matter power spectrum, which manifests itself in the concentration-mass relationship which enters the boost-factor calculation (see Ref. [21]).

\section{DISCUSSION}

There is a vast literature on galactic substructure, much of it discussing the implications for dark-matter detection. Most of this work focuses on the annihilation boost factor, but there are few papers that discuss the implications for direct detection. The primary focus of these papers is then on the effects of substructure on the local dark-matter velocity distribution, with less attention to the possible implications for the local dark-matter density. Here we review some of the work that discusses the implications of substructure on the local dark-matter density.

In Ref. [23], the authors use numerical simulations to investigate the effects of substructure on the local velocity distribution and on the local density. The conclusion of that paper is that most of the local dark matter is smoothly distributed (illustrated in their Fig. 5, which resolves structure down to $\sim 10^{7} M_{\odot}$ ). The fraction of mass that is bound in halos less massive than $10^{7} M_{\odot}$ is less than $4 \%$; this is the fraction of the mass that could have survived the tidal field of the Milky Way. They thus conclude that it is highly unlikely that a good fraction of the halo is in Earth-mass subhalos. However, their calculation does not consider the halos-in-halos problem: i.e., their mass function $d N / d m$ keeps track only of the most massive halo in which a given particle resides, not the least massive subhalo. Put another way, their calculation counts only the Earth-mass halos that did not get incorporated into more massive halos, and it disregards those that did, assuming simply that they were completely disrupted. Still, this paper does argue that substructures at later stages in the merger hierarchy get largely erased. Although isolated Earth-mass substructures may survive in a smooth Milky Way halo (as pointed out also in Ref. [22]), when scaled to earlier generations in the hierarchy, the conclusions of this paper imply that Earth-mass subhalos are likely to be disrupted when they merge into subsequent stages in the hierarchy (e.g., into $1000 M_{\oplus}$ halos). If so, then $f(\rho)$ will be very small $(f(\rho) \ll 0.2)$ ).

A local dark matter density $\operatorname{PDF} P(\rho)$ is calculated in Ref. [24], but it is a different distribution. In particular, their calculation assumes that the vast majority of the local dark matter is smoothly distributed, and that only $1 \%-5 \%$ of the local dark matter may be in substructure. The density $\rho$ that appears in their PDF is thus the density of this additional substructure component, which they assume comes from the latest subhalos accreted onto the Milky Way halo. Their mass function does extend to small masses, but they do not consider halos-in-halos. Reference [25] is similar in spirit, but considers, in particular, the effects of the tails of the Sagittarius dwarf.

The approach that most closely resembles ours is perhaps that in Ref. [22]. They recognize the scale-invariant nature of the problem - that is, that there may be halos in halos-but then note that the resolution limits of their simulation prohibits them from making definitive claims about earth-mass objects. Their calculations indicate that the singular cores of subhalos may always survive, even if most of the mass from a given subhalo is stripped. They provide as an example a subhalo orbiting at $20 \mathrm{kpc}$ in the Milky Way in which only $0.3 \%$ of the initial mass remains after four orbits, but then show that the survival fraction may be as high as $40 \%$ for an orbit at $40 \mathrm{kpc}$. The simulations of Ref. [22] make the important point that tidal tails broaden rapidly, and this justifies the assumption of our first analytic model that matter stripped from a subhalo is rapidly smoothly distributed in the new larger halo.

This assumption is also justified by the recent results presented in Ref. [33]. In this work, the authors proposed a technique for calculating the fine-grained phase space structure in dark matter halos from cosmological N-body simulations. In demonstrating the effectiveness of this new method, they studied the evolution of the dark matter density that arises from streams in NFW-like potentials, and found that a very large number of streams $\left(\sim 10^{5}\right)$ may potentially be present in the solar neighborhood. If each stream has a diameter of order $\sim \mathrm{kpc}$, it means that their entanglement and evolution has an effect in the local smooth dark matter density, and thus do not address the potential enhancement on much smaller scales.

\section{CONCLUSIONS AND CHALLENGES}

It is evident from simulations and analytic arguments that some fraction of the local Milky Way dark matter may be in subhalos. The implications of substructure for indirect detection of WIMPs have been studied broadly, with the conclusion that there may be large enhancements in annihilation rates over the rates predicted assuming a smooth halo. The implications for direct searches have, however, been largely overlooked. This is a possibly serious omission, as one consequence of substructure is that the local density will be smaller than the smoothly distributed local density usually assumed.

We have taken a few first steps to understand the implications of substructure for the local density. Our central goal is a calculation of the PDF $P(\rho)$ for the local darkmatter density $\rho$. This $P(\rho)$ will need to be taken into account when interpreting the implications of null darkmatter searches for constraints to the particle-dark-matter parameter space (e.g., couplings and/or elastic-scattering cross sections). 
We considered two simple scenarios for substructure: In the first, early generations of very dense subhalos survive with some probability. The advantage of this approach is that if subhalo survival fractions can be measured in simulations for recently merged subhalos, the results might be extrapolated to the much earlier generations (much smaller and denser subhalos) that may be below the resolution of simulations.

In the second approach, the halo is assumed to consist of recently formed subhalos, each with an NFW profile. This approach provides a simple, albeit approximate, way to understand the effects of larger-scale substructure, from more recent stages in the merger hierarchy, on the PDF. We then pursued this approach further using subhalo mass functions and concentration-parameter distributions taken from simulations. This approach does not take into account the possible contribution of subhalos within subhalos. In principle, a complete solution for the PDF, including substructures on the largest and smallest scales, can be obtained by convolving our two calculations. This, however, will be left for future research.

Substructure scenarios that yield larger annihilation enhancements generally imply a smaller local dark-matter density. Very large annihilation enhancements require that the very densest substructures, which generally form earlier, must survive through all later generations of structure formation. If earlier substructures are less likely to survive than more recent substructures, then a very large annihilation enhancement is unlikely.

So, how small can the local density be? The smallest local density in the models we surveyed was one-tenth the canonical value of $0.4 \mathrm{GeV} \mathrm{cm}^{-3}$, usually assumed for a smoothly distributed halo. This small value was obtained from our simulation-inspired result using what we believe to be an overly conservative estimate of the smooth fraction, which we obtained by truncating NFW subhalos when their density falls below the mean halo density. More realistic values for the smooth fraction are probably in the range of $50 \%-80 \%$, which would then correspond in our simulation-inspired results to the $\xi=0.8$ and $\xi=0.5$ distributions, respectively, shown in Fig. 3. The smallest local density in our analytic model for early substructure was 0.3 , obtained using the high value, $f\left(\rho_{\odot}\right)=0.2$, and assuming a constant $f\left(\rho_{1}\right)$. If, however, $f\left(\rho_{\odot}\right)$ is lower, then the local density is increased. More importantly, it is quite likely that $f(\rho)$ decreases with $\rho$, and if so, the local density is increased to $\sim 0.8$ times its canonical value, even for $f\left(\rho_{\odot}\right)=0.2$, and even higher for smaller $f\left(\rho_{\odot}\right)$. A combination of our two approaches, to take into account both low- and high-density substructures, will likely show that the local density is no less than half the canonical value.

The volume of the halo probed during a three-year direct-detection experiment is very small, and so the halo density $\rho$ that we have been discussing can be safely assumed to be the density averaged over the duration of a direct-detection experiment. However, the rate for production of energetic neutrinos from WIMP annihilation in the Sun or Earth lags behind the rate for capture of WIMPs from the halo by an equilibration time $t_{\mathrm{eq}}[34,35]$ that can vary considerably with the WIMP's mass and elasticscattering and annihilation cross sections; typical values might be $t_{\text {eq }}^{\odot} \sim 5 \times 10^{7} \mathrm{yr}$ and $t_{\text {eq }}^{\oplus} \sim 10^{10} \mathrm{yr}[36]$. An energetic-neutrino search thus probes the halo density averaged over a much larger volume (for the latest bounds on the flux of high-energy neutrinos from annihilations in the Earth and the Sun see [37-39]). The halo density averaged over this volume will thus have a PDF that will be narrower than that for direct detection, and the minimum density will be closer to the mean halo density. However, even though initially it was assumed that the velocity distribution function of dark matter particles mirrors that in free space (thus justifying the use of a MaxwellBoltzmann distribution) [40], it is possible that the velocity distribution function is suppressed in the low-velocity tail due to the effects of solar capture and WIMP diffusion in the solar system due to the presence of other planets $[41,42]$. This suppression manifests itself as a reduction in the number density of dark matter particles near the Earth for WIMPs with masses greater than a few hundred $\mathrm{GeV}$. Nevertheless, there is a possibility that for low-mass WIMPs, given the longer equilibration time for the Earth relative to that for the Sun, the annihilation signal from the Earth could be boosted relative to that for the Sun, if the Solar System passed through a very dense subhalo at a time $t_{\text {eq }}^{\odot}<t<t_{\text {eq }}^{\oplus}$ ago. We leave a more detailed calculation of the density smoothed over time scales relevant for energetic-neutrino searches to future work [43].

The PDF $P(\rho)$ allows us to evaluate also the boost factor in the annihilation rate. It must be emphasized, though, that the boost factor $B$ we have considered is the boost only in the local annihilation rate (per unit volume). Strictly, speaking, the PDF may vary from one position in the halo to another. Thus, for example, if we assume that the halo is spherically symmetric (after averaging over substructure fluctuations), then the PDF will be a function $P(\rho ; r)$ of Galactocentric radius $r$, as well as the density $\rho$. The mean density of this distribution, as a function of $r$, will be the density of the smooth NFW profile that best fits the rotation curve. Qualitatively, we expect that the highdensity tail will be more pronounced (more substructure) at larger $r$ and less pronounced (less substructure) at smaller $r$. The PDF $P(\rho ; r)$ can then be used to determine the boost factor as a function of position in the halo; again, we expect $B(r)$ to vary with $r$ and to generally increase with $r$. This $B(r)$ will be essential to compute annihilation fluxes along various lines of sight through the Galaxy. We encourage future authors to describe the effects of substructure in terms of $P(\rho ; r)$, or at least in terms of $B(r)$, as this may facilitate comparison between the conclusions of different studies. 


\section{ACKNOWLEDGMENTS}

We acknowledge useful conversations with A. Benson, S. Habib, K. Heitmann, G. Jungman, D. Nagai, and D. Reed. SMK thanks Caltech for hospitality while this work was being conceived. Work at LANL was carried out under the auspices of the NNSA of the U.S. Department of
Energy at Los Alamos National Laboratory under Contract No. DE-AV52-06NA25396. This work was supported at Caltech by DoE DE-FG03-92-ER40701, NASA NNG05GF69G, and the Gordon and Betty Moore Foundation.
[1] G. Jungman, M. Kamionkowski, and K. Griest, Phys. Rep. 267, 195 (1996).

[2] G. Bertone, D. Hooper, and J. Silk, Phys. Rep. 405, 279 (2005).

[3] D. Hooper and S. Profumo, Phys. Rep. 453, 29 (2007).

[4] M.S. Turner, Phys. Rep. 197, 67 (1990); G. G. Raffelt, Phys. Rep. 198, 1 (1990).

[5] L. J. Rosenberg and K. A. van Bibber, Phys. Rep. 325, 1 (2000); S. J. Asztalos, L. J. Rosenberg, K. van Bibber, P. Sikivie, and K. Zioutas, Annu. Rev. Nucl. Part. Sci. 56, 293 (2006).

[6] http://lhc.web.cern.ch/lhc.

[7] J. F. Navarro, C. S. Frenk, and S. D. M. White, Astrophys. J. 490, 493 (1997)

[8] S. Ghigna, B. Moore, F. Governato, G. Lake, T. Quinn, and J. Stadel, Mon. Not. R. Astron. Soc. 300, 146 (1998); A. A. Klypin, A. V. Kravtsov, O. Valenzuela, and F. Prada, Astrophys. J. 522, 82 (1999); A. A. Klypin, S. Gottlöber, A. V. Kravtsov, and A. M. Khokhlov, Astrophys. J. 516, 530 (1999); B. Moore, S. Ghigna, F. Governato, G. Lake, T. Quinn, J. Stadel, and P. Tozzi, Astrophys. J. 524, L19 (1999); V. Berezinsky, V. Dokuchaev, and Y. Eroshenko, Phys. Rev. D 77, 083519 (2008); A. Loeb and M. Zaldarriaga, Phys. Rev. D 71, 103520 (2005); E. Bertschinger, Phys. Rev. D 74, 063509 (2006).

[9] C. Boehm, P. Fayet, and R. Schaeffer, Phys. Lett. B 518, 8 (2001).

[10] A. M. Green, S. Hofmann, and D. J. Schwarz, Mon. Not. R. Astron. Soc. 353, L23 (2004); A. M. Green, S. Hofmann, and D. J. Schwarz, in The 11th International Symposium on Particles, Strings and Cosmology, edited by K. Choi, J. E. Kim, and D. Son, AIP Conf. Proc. No. 805 (AIP, New York, 2006), p. 431; A. M. Green, S. Hofmann, and D. J. Schwarz, J. Cosmol. Astropart. Phys. 08 (2005) 003.

[11] J. Diemand, B. Moore, and J. Stadel, Nature (London) 433, 389 (2005).

[12] J. Diemand, M. Kuhlen, and P. Madau, Astrophys. J. 667, 859 (2007).

[13] S. Ando and E. Komatsu, Phys. Rev. D 73, 023521 (2006).

[14] X.1. Chen, M. Kamionkowski, and X.m. Zhang, Phys. Rev. D 64, 021302 (2001).

[15] S. Profumo, K. Sigurdson, and M. Kamionkowski, Phys. Rev. Lett. 97, 031301 (2006).

[16] W. Hu, R. Barkana, and A. Gruzinov, Phys. Rev. Lett. 85, 1158 (2000).

[17] L. A. Boyle, R. R. Caldwell, and M. Kamionkowski, Phys. Lett. B 545, 17 (2002).
[18] L. Bergstrom, J. Edsjo, P. Gondolo, and P. Ullio, Phys. Rev. D 59, 043506 (1999); L. Bergstrom, J. Edsjo, and P. Ullio, Phys. Rev. D 58, 083507 (1998); C. CalcaneoRoldan and B. Moore, Phys. Rev. D 62, 123005 (2000); A. Tasitsiomi, J. M. Siegal-Gaskins, and A. V. Olinto, New Astron. Rev. 48, 473 (2004); A. Tasitsiomi and A. V. Olinto, Phys. Rev. D 66, 083006 (2002); F. Stoehr, S. D. M. White, V. Springel, G. Tormen, and N. Yoshida, Mon. Not. R. Astron. Soc. 345, 1313 (2003); S. M. Koushiappas, A. R. Zentner, and T. P. Walker, Phys. Rev. D 69, 043501 (2004); E. A. Baltz, J. E. Taylor, and L. L. Wai, arXiv:astro-ph/0610731; L. Pieri, E. Branchini, and S. Hofmann, Phys. Rev. Lett. 95, 211301 (2005); S. M. Koushiappas, Phys. Rev. Lett. 97, 191301 (2006); L. Pieri, G. Bertone, and E. Branchini, arXiv:0706.2101; J. Diemand, M. Kuhlen, and P. Madau, Astrophys. J. 657, 262 (2007); V. Berezinsky, V. Dokuchaev, and Y. Eroshenko, J. Cosmol. Astropart. Phys. 07 (2007) 011; Phys. Rev. D 68, 103003 (2003).

[19] C. Giocoli, L. Pieri, and G. Tormen, arXiv:0712.1476.

[20] V. Berezinsky, V. Dokuchaev, and Y. Eroshenko, Phys. Rev. D 73, 063504 (2006).

[21] L. E. Strigari, S. M. Koushiappas, J. S. Bullock, and M. Kaplinghat, Phys. Rev. D 75, 083526 (2007).

[22] B. Moore, C. Calcaneo-Roldan, J. Stadel, T. Quinn, G. Lake, S. Ghigna, and F. Governato, Phys. Rev. D 64, 063508 (2001).

[23] A. Helmi, S. D. M. White, and V. Springel, Phys. Rev. D 66, 063502 (2002).

[24] D. Stiff, L. M. Widrow, and J. Frieman, Phys. Rev. D 64, 083516 (2001).

[25] K. Freese, P. Gondolo, and H. J. Newberg, Sagittarius Phys. Rev. D 71, 043516 (2005).

[26] A. Benson (private communication).

[27] H. Zhao, J. E. Taylor, J. Silk, and D. Hooper, Astrophys. J. 654, 697 (2007); H. S. Zhao, J. Taylor, J. Silk, and D. Hooper, arXiv:astro-ph/0502049; T. Goerdt, O. Y. Gnedin, B. Moore, J. Diemand, and J. Stadel, Mon. Not. R. Astron. Soc. 375, 191 (2007).

[28] D. Reed, F. Governato, T. Quinn, J. Gardner, J. Stadel, and G. Lake, Mon. Not. R. Astron. Soc. 359, 1537 (2005).

[29] J. Diemand, B. Moore, and J. Stadel, Mon. Not. R. Astron. Soc. 352, 535 (2004).

[30] J. Diemand, M. Kuhlen, and P. Madau, Astrophys. J. 649, 1 (2006).

[31] J. S. Bullock et al., Mon. Not. R. Astron. Soc. 321, 559 (2001).

[32] R.H. Wechsler, J.S. Bullock, J.R. Primack, A. V. 
Kravtsov, and A. Dekel, Astrophys. J. 568, 52 (2002).

[33] M. Vogelsberger, S.D.M. White, A. Helmi, and V. Springel, arXiv:0711.1105.

[34] K. Griest and D. Seckel, Nucl. Phys. B283, 681 (1987); B296, 1034(E) (1988).

[35] M. Kamionkowski, Phys. Rev. D 44, 3021 (1991).

[36] M. Kamionkowski, K. Griest, G. Jungman, and B. Sadoulet, Phys. Rev. Lett. 74, 5174 (1995).

[37] S. Desai et al. (Super-Kamiokande Collaboration), Phys. Rev. D 70, 083523 (2004); 70, 109901(E) (2004).
[38] A. Achterberg et al. (AMANDA Collaboration), Astropart. Phys. 26, 129 (2006).

[39] M. Ackermann et al. (AMANDA Collaboration), Astropart. Phys. 24, 459 (2006).

[40] A. Gould, Astrophys. J. 368, 610 (1991).

[41] A. Gould and S. M. Khairul Alam, Astrophys. J. 549, 72 (2001).

[42] J. Lundberg and J. Edsjo, Phys. Rev. D 69, 123505 (2004).

[43] M. Kamionkowski and S. Koushiappas (unpublished). 